\title{
Model Dispersion Analysis of Circular Waveguide in Normal and Reverse Boundary Condition
}

\author{
N. Shamini, K. Deepak , Y.N Phua
}

\begin{abstract}
The dispersion characteristics of the circular step index fiber with helical windings between the core-cladding region is investigated. Sheath helix is wounded between the core and cladding using two directions, namely in the clock wise and anticlockwise direction. Substituting the field components into the modified boundary conditions due to the addition of the helical windings the modal characteristics are derived for both fibers. Representations of the helical windings are done by using normal boundary conditions and reverse boundary conditions. The Eigen equation is obtained in the form of Bessel functions and modified Bessel functions for both the waveguides. The dispersion curves are plotted for two specific pitch angles $\psi=0^{\circ}$ and $\psi=90$ for each fiber and the results are compared. The direction of wrapping the helical material results in a change in the dispersion properties with regards to the way the modes propagate in both fibers. These changes are seen by the presents and absence of (1) band gap,(2) splitting of modes and (3) adjacent modes depending on the direction and pitch angle of the helical windings. Results obtained in this work suggest that direction and pitch angle of the helical winding are parameters that are able to control the behavior of the modes. .
\end{abstract}

Index Terms: Bessel function, circular step index fiber, helical winding, dispersion characteristic

\section{INTRODUCTION}

Analysis of the propagation characteristics of optical fiber is an area that has been of interest for various optical geometries such as rectangular, cylindrical and elliptical [1-5].The helical winding that utilizes sheath helix was later introduced between the core and cladding boundary of the optical fiber [6].The study of helical winding in optical fiber is motivated from its application in the traveling wave tubes .The helical structure is commonly used in all low to medium power travelling wave tubes and also in oscillators such as the low power backward wave oscillators [8]. Pioneering work was first done on the step index fiber circular waveguides with helical winding [6] which are known as circular helically cladded fibers(HCF), this work was then further extended to the elliptical geometry [7]. All

Revised Manuscript Received on August 18, 2019

Shamini Pillay, Faculty of Engineering, Multimedia University Cyberjaya ,Malaysia

Deepak Kumar, Faculty of computing and informatics Multimedia University Cyberjaya Malaysia.

Phua Yeong Nan, Department of Electrical and Electronic Engineering Universiti Tunku Abdul Rahman (UTAR), Kajang, Malaysia. previous works [6-9] used normal boundary conditions with various geometries to determine the Eigen equations. Dispersion characteristics were analyzed for those works as they described the behavior of modes propagating in the fiber. In the present work we have done a comparative study of the circular step-index fiber with helical winding using reverse boundary condition and normal boundary condition to obtain the Eigen equation and further plot the dispersion curves. Previously, we have completed the mathematical derivation for both reverse and normal boundary conditions. Details of the derivation are found in [10] and [16] respectively.

The specific taken pitch angles are namely $\psi=0^{\circ}$ and $\psi=90^{\circ}$ for the fibers. These pitch angles are the angles in which the sheath helix is wrapped between the core and cladding. We have simulated for these specific angles as they have shown to give interesting behavior of modes in [9] for the elliptical and circular geometry. The realization of sheath helix in optical fiber and the boundary conditions are described in the work of Watkins [15]. Implementation of the sheath helix between the core and cladding in practice can be of many similar windings that are tightly bound side by side but insulated from each of the windings. Alternatively, a thin planer sheet made of alternate of conducting and non-conducting gaps are wrapped around the cylindrical core without overlap. Both of the implementation are shown and discussed in detail in [9]. The main idea of the current work is to compare the behavior of modes for the cylindrical step index fiber with helical windings and determine if the direction of windings has any impact on the behavior of the propagating modes.

Mathematical complexity in analyzing fibers are usually difficult under strong guidance condition [11] [12] due to the difference between reflective indexes for the core and cladding being large. Thus weak guidance is used for our analysis whereby the difference between the reflective indexes for the core and cladding are kept very small. The Eigen equation obtained is of the Bessel and modified Bessel functions [13]. By using this equation, dispersion curves are plotted in both cases to compare the behavior of the modes. Fig-1 shows both our fibers used in this work. 

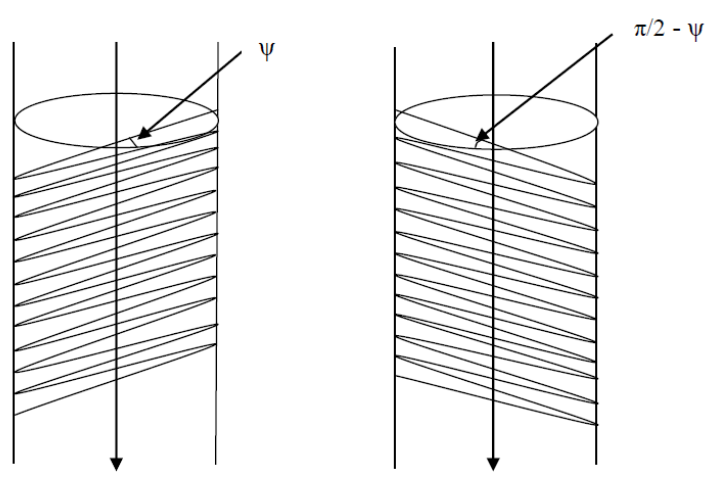

Fig. 1. Circular step index fiber with helical windings between core and cladding

\section{FIELD COMPONENTS}

The electric and magnetic field in the core and cladding of the fiber are represented by the following eight equations [13][14]. Table 1 shows the boundary conditions used to obtain the Eigen equation for both fibers. The axial electric and magnetic field components can be expressed as:

For core region $(\mathrm{r}<\mathrm{a})$

$$
E z_{1}=\mathrm{A} . \mathrm{J}_{v}(u a) e^{i(\omega t-\beta z+v \phi)}
$$

$$
\begin{aligned}
& H z_{1}=\text { B.J } v_{v}(u a) e^{i(\omega t-\beta z+v \phi)} \\
& E_{\phi 1}=-\frac{i}{u^{2}}\left[\frac{i v \beta}{a} A J_{v}(u a)-\omega \mu_{0} u B J_{v}^{\prime}(u a)\right] e^{i(\omega t-\beta z+v \phi)} \\
& H_{\phi 1}=-\frac{i}{u^{2}}\left[\frac{i v \beta}{a} B J_{v}(u a)+\omega \varepsilon_{1} A u B J_{v}^{\prime}(u a)\right] e^{i(\omega t-\beta z+v \phi)}
\end{aligned}
$$

For cladding region $(\mathrm{r}>\mathrm{a})$

$$
E z_{2}=\mathrm{C} \cdot \mathrm{K}_{v}(w a) e^{i(\omega t-\beta z+v \phi)}
$$

$$
H z_{2}=\mathrm{D} \cdot \mathrm{K}_{v}(w a) e^{i(\omega t-\beta z+v \phi)}
$$

$$
E_{\phi 2}=-\frac{i}{w^{2}}\left[\frac{i v \beta}{a} C K_{v}(w a)-\omega \mu_{0} w D K_{v}^{\prime}(w a)\right] e^{i(\omega t-\beta z+v \phi)}
$$

$$
H_{\phi 2}=-\frac{i}{w^{2}}\left[\frac{i v \beta}{a} D K_{v}(w a)+\omega \varepsilon_{2} C w K_{v}^{\prime}(w a)\right] e^{i(\omega t-\beta z+v \phi)}
$$

Substituting the value of $E_{z 1},{ }_{z 1},{ }_{z 2},{ }^{H}{ }_{z 2},{ }^{H}{ }_{\phi 1},{ }^{E_{\phi 2}}$ and $H_{\phi 2}$ from equations(1-8) into both the boundary condition equations shown in Table-1, four equations with four constants A,B,C and D respectivily for both the boundary condition are formed. Eliminating the constants $\mathrm{A}, \mathrm{B}, \mathrm{C}$ and $\mathrm{D}$ from the equations a matrix of order of $(4 \mathrm{x} 4)$ is obtained. After expanding the determinant, we have an equation in terms of Bessel function and modified Bessel functions for both the boundary condition. Details of the derivation can be seen in our previous works [16]and [10] respectively.

Table 1. Boundary condition used for normal and reverse case [10]

\begin{tabular}{|l|l|}
\hline \multicolumn{1}{|c|}{$\begin{array}{c}\text { Boundary Condition } \\
\text { (Normal) }\end{array}$} & \multicolumn{1}{c|}{$\begin{array}{c}\text { Boundary Condition } \\
\text { (Reverse) }\end{array}$} \\
\hline$E_{z 1} \sin \psi+E_{\phi 1} \cos \psi=0$ & $E_{z 1} \cos \psi-E_{\phi 1} \sin \psi=0$ \\
$E_{z 2} \sin \psi+E_{\phi 2} \cos \psi=0$ & $E_{z 2} \cos \psi-E_{\phi 2} \sin \psi=0$ \\
$E_{z 1} \cos \psi-E_{\phi 1} \sin \psi=E_{z 2} \cos \psi-E_{\phi 2} \sin \psi$ & $E_{z 1} \sin \psi+E_{\phi 1} \cos \psi=E_{z 2} \sin \psi+E_{\phi 2} \cos \psi$ \\
$H_{z 1} \sin \psi+H_{\phi 1} \cos \psi=H_{z 2} \sin \psi+H_{\phi 2} \cos \psi$ & $H_{z 1} \cos \psi-H_{\phi 1} \sin \psi=H_{z 2} \cos \psi-H_{\phi 2} \cos \psi$ \\
& \\
\hline
\end{tabular}

\section{NUMERICAL RESULTS}

A representative numerical computation is able to illustrate the use of the Eigen equations that were derived in our previous works [10][16]. This is done by taking values of $\psi$ as $0^{\circ}$ and $90^{\circ}$. For the computations we have to refer to the core parameter, ua, where $u=\left(\mathrm{k}^{2} n_{1}^{2}-\beta^{2}\right)^{1 / 2}$. Two other parameters are the cladding parameter wa, where $w=\left(\beta^{2}-\mathrm{k}^{2} n_{2}^{2}\right)^{1 / 2}$ and the normalised frequency, that is, the V-parameter, where $V=\frac{2 \pi \mathrm{a}}{\lambda_{0}}\left(\mathrm{n}_{1}^{2}-\mathrm{n}_{2}^{2}\right)^{1 / 2}$. The normalised propagation constant is denoted as $b_{n o r}$.

The normalised propagation constant $b_{\text {nor }}$ is defined as:

$$
b_{\text {nor }}=\left\{\frac{\beta^{2}-\mathrm{k}_{0}^{2} n_{2}^{2}}{\mathrm{k}_{0}^{2}\left(n_{1}^{2}-n_{2}^{2}\right)}\right\}^{1 / 2}
$$

In our computations we have chosen $\mathrm{n}_{1}$ as $1.50, \mathrm{n}_{2}$ as 1.46 and the free space operating wavelength $\lambda$ is chosen at 1.5 $\mu \mathrm{m}$, as commonly used in fibre optics. The algorithm used is as follows; fix a $\mathrm{V}$ parameter and take numerous equal spaced values of $\beta$ in the range $n_{1} k<\beta<n_{2} k$. The zero-crossing values of $\beta$ are then tabulated. These values represent the possible $\beta$-values for the sustained modes for a given value of $\mathrm{V}$. 
The procedure is then repeated for several values of $\mathrm{V}$ ranging from 0 to 72 . If we now choose a given mode and plot the possible $\beta$-values (or the normalised propagation parameter $b_{\text {nor }}$ ) against the V-parameter, we find a curve representing the dispersion properties of the modes. In this work, five lower order modes are simulated as seen in the dispersion curves. Fig-2[i-x] and Fig-3[i-x] represents th dispersion curve for normal boundary condition and rever: boundary condition respectively. Four behaviours of th modes are observed and analysed using the dispersion curvi namely band gaps, number of modes at $\mathrm{V}<7$, splitting an adjacent modes.

The peculiarity in the normal boundary condition can $t$ observed in Fig. 2(i-v) where the pitch angle $\psi=0^{\circ}$, the: peculiarities are not seen in normal fibres. There are for band gaps in Fig. 2(i), two bandgaps in Fig. 2(ii), three banu gaps in Fig. 2(iii) and one band gap in Fig. 2(iv-v). In these band gaps we are unable to see any portion of the dispersion curves. These portions are called forbidden regions. Whereas for pitch angle $\psi=90^{\circ}$ in Fig. 2(vi-x) there are no band gaps observed.

For the reverse boundary condition Fig. 3(i-x) for both pitch angles there are no band gaps. In our Previous work done [10] for the reverse boundary condition we had obtained band gaps for the various pitch angles namely $10^{\circ}, 20^{\circ}, 30^{\circ}$. Due to using similar parameters except for the pitch angle, we conclude that the change in pitch angle is one of the factors contributing to the band gaps observed when implementing the reverse boundary condition.

Next we look at the number of propagating modes that are present when $\mathrm{V}<7$. For the reverse boundary condition where $\psi=0^{\circ}$ we observe that there are 5 modes for Fig. 3(i) ,3 modes for Fig. 3( ii-iii) and in Fig. 3(iv-v) the number of modes are 1. This is the exact number of modes that are obtained for the normal boundary condition in Fig. 2(vi-x) whereby $\psi=90^{\circ}$. For the reverse boundary condition where $\psi=90^{\circ}$ we are able to see from Fig. 3(vi-x); the number of modes are 4 in Fig. 3 (vi) and the number of modes are 3 and 2 in Fig. 3(vii) and Fig. 3(viii) respectively, whereas the number of mode is 1 in Fig. 3(ix-x). The number of modes obtained for normal boundary condition where $\psi=0^{\circ}$ is shown in Fig. 2(i-v). Here in Fig. 2(i-v) it shows that the number of modes propagating is same as seen in the reverse case for $\psi=90^{\circ}$. According to the results obtained we are able to deduce that as the modal index number $v$ is increasing the number of modes propagating are decreasing at $\mathrm{V}<7$ for both pitch angles and also in both cases of normal and reverse boundary condition. The similarity of both the cases is the reduction of modes at $\mathrm{V}<7$. But we notice that this reduction is more consistent in reverse boundary condition for pitch $\psi=90^{\circ}$ and in the normal case for $\psi=0^{\circ}$.

Next we observe that adjacent and splitting modes are seen for both the normal and reverse case. Referring to
Fig. 2(i-v) for normal boundary condition for $\psi=0^{\circ}$ it is observed that we get splitting of modes whereas in Fig. 2(vi-x) for $\psi=90^{\circ}$ there are adjacent pairs of modes . In the reverse boundary condition Fig. $3(\mathrm{i}-\mathrm{x})$ for $\psi=0^{\circ}$ adjacent modes are obtained and for $\psi=90^{\circ}$ splitting of

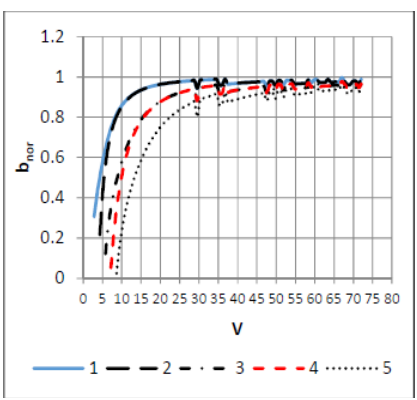

(i) $v=1, \psi=0^{\circ}$

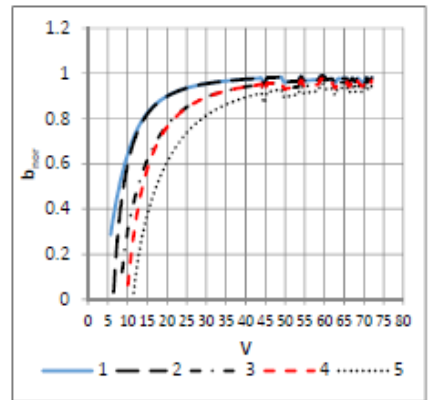

(iii) $v=3, \psi=0^{\circ}$

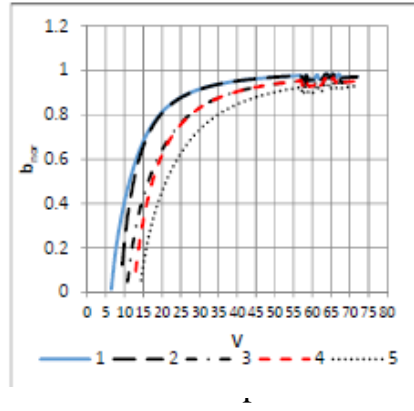

(v) $v=5, \psi=0^{\circ}$

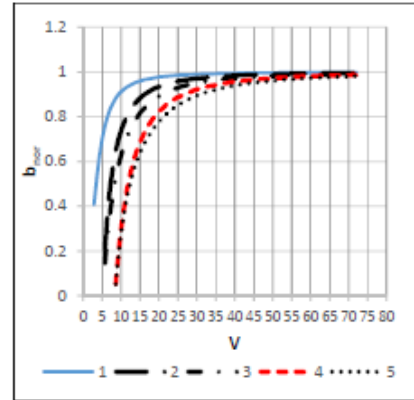

(vii) $v=2, \psi=90^{\circ}$

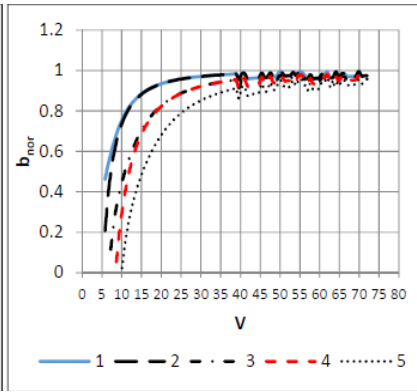

(ii) $v=2, \psi=0^{\circ}$

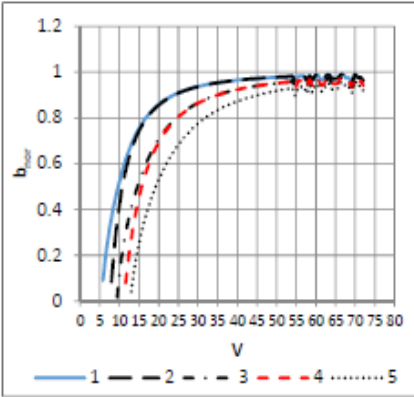

(iv) $v=4, \psi=0^{\circ}$

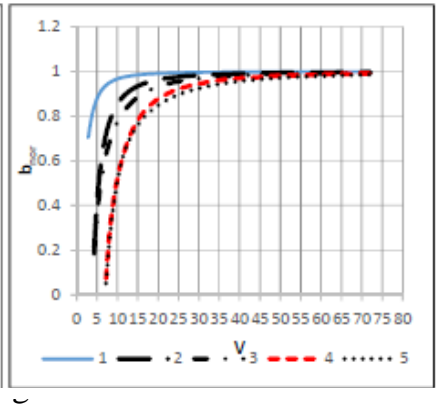

(vi) $v=1, \psi=90^{\circ}$

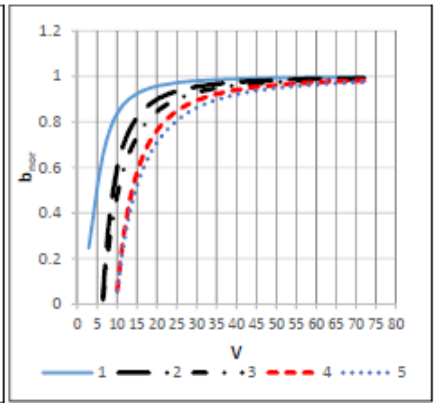

(viii) $v=3, \psi=90^{\circ}$ 


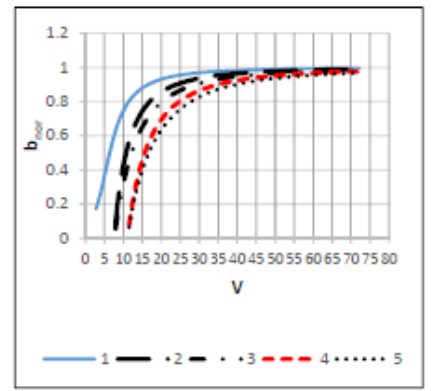

(ix) $v=4, \quad \psi=90^{\circ}$

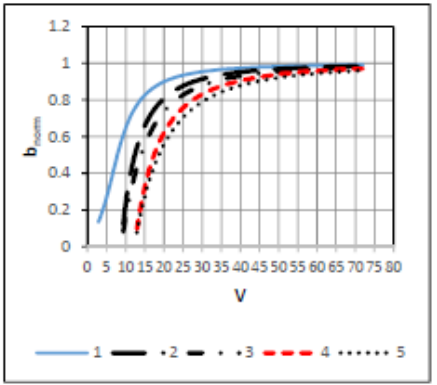

(x) $v=5, \psi=90^{\circ}$
Fig. 2(i-x) Dispersion curve for normalised propagation constant $\left(b_{\text {nor }}\right)$ verses normalised frequency $(V)$ for normal boundary condition.

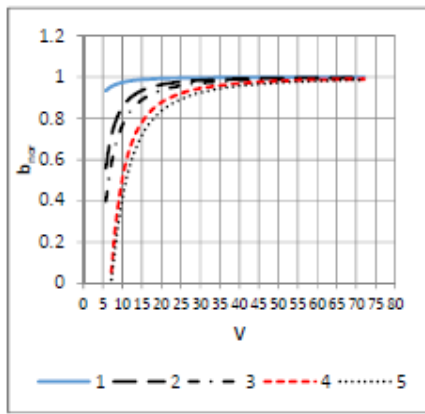

(i) $v=1, \psi=0^{\circ}$

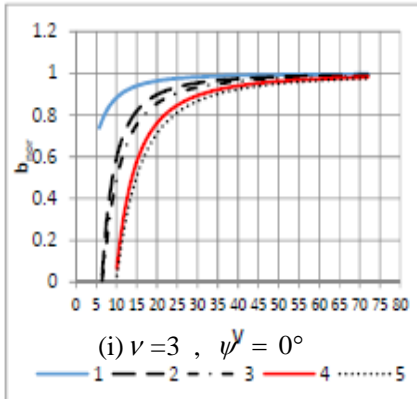

(i) $v=3, \psi=0^{\circ}$

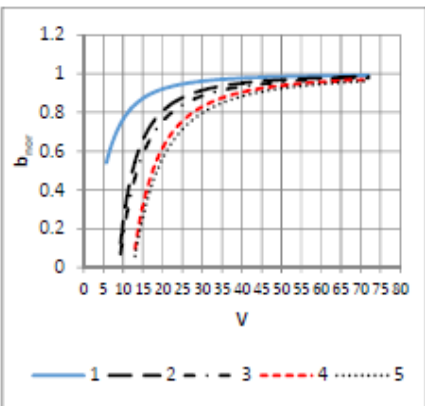

(v) $v=5, \psi=0^{\circ}$

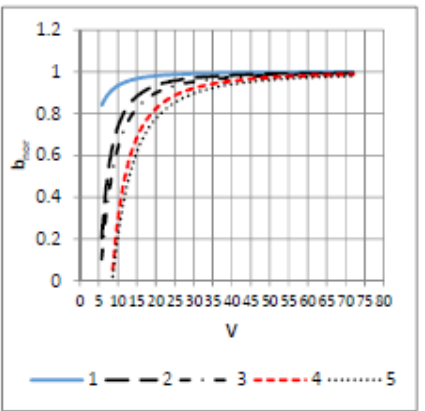

(ii) $v=2, \psi=0^{\circ}$

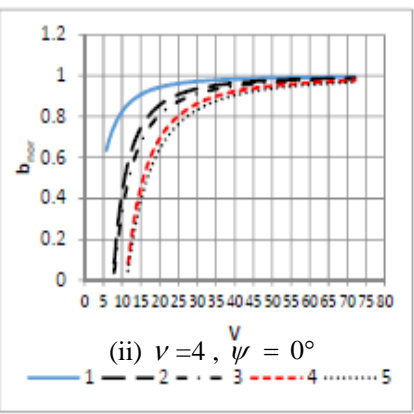

(ii) $v=4, \psi=0^{\circ}$

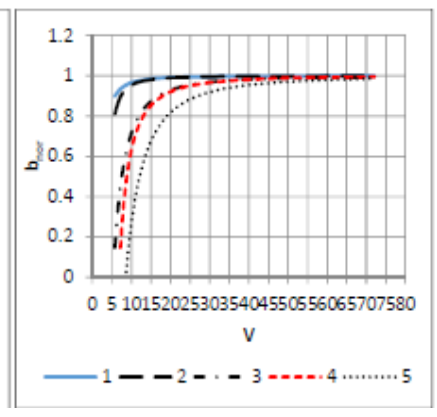

(vi) $v=1, \psi=90^{\circ}$

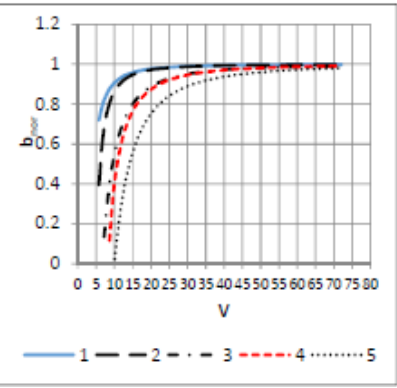

(vii) $v=2, \psi=90^{\circ}$

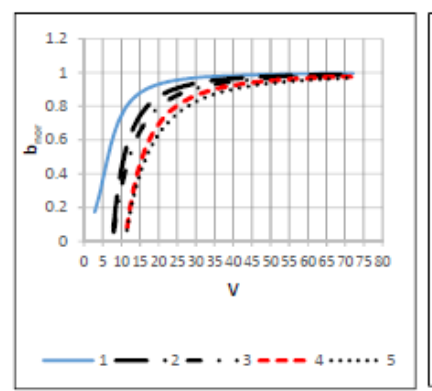

(ix) $v=4, \psi=90^{\circ}$

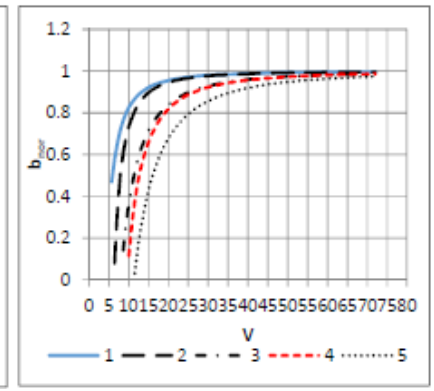

(viii) $v=3, \psi=90^{\circ}$

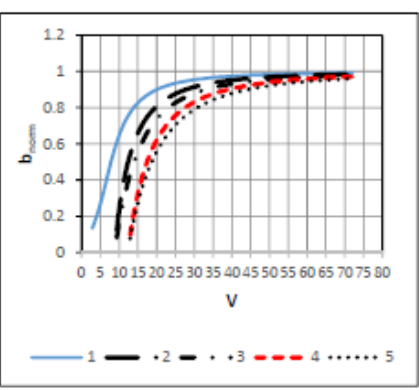

(x) $v=5, \psi=90^{\circ}$
Fig. 3(i-x) Dispersion curve for normalised propagation constant $\left(b_{\text {nor }}\right) \quad$ verses normalised frequency $(V)$ for reverse boundary condition.

\section{CONCLUSION}

A comparative study was carried out for the circular waveguide with helical windings between the core and cladding parameters. This work is an extension and refinement to our previous work [10][16] which considered only a few pitch angles namely $10^{\circ}, 20^{\circ}, 30^{\circ}$. The dispersion curves for those angles showed limited amount of mode behaviour. Thus extending the work to cover the two specific helical pitch angles $\psi=0^{\circ}$ and $\psi=90^{\circ}$, these pitch angles have previously shown distinctive dispersion curves in previous work [9]. Two direction of winding were employed namely in the clockwise and antilock wise direction. The normal and reverse boundary condition are used to represent the two direction respectively. By substituting field components into boundary conditions, the eigen equation is obtained and then the dispersion curves are plotted. From the plots, four types of mode behaviour are observed namely band gaps, number of modes at $\mathrm{v}<7$, splitting of modes and adjacent modes.

There are some pecularites noted for the normal boundary condition, whereby band gaps are present when $\psi=0^{\circ}$. These types of band gaps are not ususally present in normal fibers without the helix. The number of band gaps also reduced as the modal index number $v$ is increased from 1 to 5. For $\psi=90^{\circ}$ band gaps are not present. 
In the reverse boundary condition there is no band gap for both pitch angles. Our Previous work done on reverse boundary condition showed band gap for the pitch angles $10^{\circ}, 20^{\circ}, 30^{\circ}$ [10]. Since similar parameters were used in the previous and current work with exception of the pitch angles, thus it may be concluded that the pitch angle is one of the factors contributing to the band gaps.

The number of modes were observed for both boundary conditions at $\mathrm{V}<7$. The number of modes in both cases decreases as the modal index increases. It is observed that the reduction in modes is similar in number for the case of normal boundary condition where $\psi=0^{\circ}$ and $\psi=90^{\circ}$ with the reverse boundary condition where $\psi=90^{\circ}$ and $\psi=0^{\circ}$ respectively. This behaviour is probably due to the wrapping direction of helix which makes the pitch angles the exact opposite of each other, as shown in Fig. 1.

Lastly we looked at adjacent and splitting modes for both the normal and reverse case. For normal boundary condition for $\psi=0^{\circ}$ it is noticed that splitting of modes occur and for $\psi=90^{\circ}$ there are adjacent pairs of modes. Using the reverse boundary condition, characteristics of these modes are seen to be reversed. Splitting of modes occurs at $\psi=90^{\circ}$ and adjacent occurs at $\psi=0^{\circ}$. The change in boundary condition is the most probable factor that a change in mode behavior is observed

To conclude, the pitch angle and direction of the helix are the probable parameter that has brought forward the mode behaviour discussed in this work. Apart from this, the forbidden region obtained when using helix might have some technological importance in fabricating filters as the modes display properties of band gap. Further by using different pitch angles of the helix the range of the band gap can be controlled. Thus it would be possible to use these additional parameters namely the direction and pitch angle of the helical windings to increase effectiveness of future application in the optic and also the optoelectronics field.

\section{REFERENCES}

1. Arun Kumar, K. Thyagarajan and A. K. Ghatak,"Analysis Of Rectangular Core Dielectric Waveguides: An Accurate Perturbation Approach,” Optics Letters (USA), 8, pp.63-65 January 1983.

2. E. Snitzer "“Cylindrical dielectric waveguide modes," J. Opt. Soc. (NA), vol. 51, pp. 491-498, May 1961.

3. C.Yeh, "Guided-wave modes in cylindrical optical Fibers," IEEE Transaction On Education", Vol.E-30,No.1,February 1987.

4. C.Yeh,"Modes in weakly guiding elliptical fibers,"Opt. Quantum Electron.," Vol. 8,pp43-47, 1976.

5. R.B Dyott: Cutoff of the first order modes in elliptical dielectric waveguide,an experimental approach," Electron. Lett., Vol. 26 , 1721-1723, 1990.

6. U.N.Singh, O.N.SinghII," P.Khastgir,and K.K.Dey,Dispersion characteristics of a helically cladded step-index optical fiber An analyticastudy,"J.Opt.Soc.Amer.B,Opt.Phys.,vol.12,pp.1273-1278,July 1995.

7. Deepak Kumar and O.N.SinghII, "Modal characteristic equation and dispersion curves for an elliptical step-index fiber with a conducting helical winding on the core-cladding," IEEE J. Lightwave Tech. (U.S.A) 20, 1416-1424 (2002)

8. J.R.Pierce,“ Travelling wave tubes," Van Nostrand company,New-York, 1950 .

9. D.Kumar and O.N.SinghII, "Elliptical and Circular step-index fibers with conducting Helical windings on the core-cladding boundaries for different pitch angles- A comparative modal dispersion analysis,"Progress in Electromagnetic Research,PIER52,1-21,2005.

10. Shamini Pillay,Deepak Kumar,Y.N.Phua and Hairul Azhar,“ Analysis of the propagation characteristics of circular step-index fiber with new sheath helix boundary condition," Journal of Electromagnetic Waves and Applications, 1-10,Dec-2013.

11. A.W.Snyder,"Asymptotic expressions for eigenfunctions and eigenvalues of a dielectric optical waveguide,".IEEE Trans.Microw.Theory Tech.17(1969),page1130-1138

12. D.Gloge,“Weakly guiding fibers,”Appl.opt.10(1971)2252-2258

13. A.H.Cherin, "An Introduction to optical fibers," pp.85-98,McGraw-Hill,’New-York1987

14. G.Keiser,"Optical Fiber Communication,"McGraw-Hill,New-York 2011.

15. D.A.Watkins, "Topics in Electromagnetic Theory," John Wiley and Sons, Inc..

16. Shamini Pillay, Deepak Kumar,Y.N.Phua,"Comparative study of boundary condition with helix,” Proc SPIE 10150, Second International Seminar on Photonics, Optics, and its Application(ISPhOA 2016),1015006, November 11,2016.

\section{AUTHORS PROFILE}

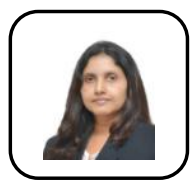

Ms. Shamini Pillay received her B.Eng. (Hons) degree in Computer \& Communications Systems and M.S degree in Communications and Networking Engineering from University Putra Malaysia, in 2001 and 2004. She has been lecturing till present in the Faculty of Engineering at Multimedia University Malaysia since 2008. Currently she is completing her Ph.D. thesis in the field of fiber optics. She has authored 5 International journals. Her research interests include optical fibers, applied electromagnetics and wireless networks.

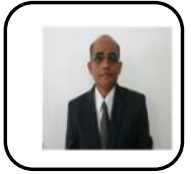

Dr. Deepak Kumar received his Ph.D. degree in applied physics from the Institute of Technology, Banaras Hindu Uinversity, Varanisi, India, in 1999. From 2001-2004, he was a lecturer in physics with the U.N.S. Institute of Engineering and Technology, Jaunpur, India. Later from 2005-2018 he was a Senior Lecturer in Faculty of Engineering Multimedia University Cyberjaya Malaysia. Presently he is working as a Senior Lecturer Faculty of computing and informatics Multimedia University Cyberjaya Malaysia. He has 24 international Journal Publications and co-author of 4 books. His research interests include optical fibers and applied electromagnetics.

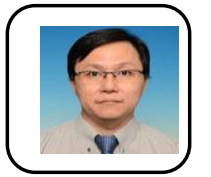

Mr. Phua Yeong Nan received his B.S. degree in Physics and M.S degree in Optoelectronics from University of Malaya, Malaysia, in 1998 and 2003, respectively. During his postgraduate study, he worked as a research assistant at the Institute of Advanced Studies. Later, he joined Multimedia University as a lecturer and carried out fiber optics related research. $\mathrm{He}$ also went into the manufacturing industry as an optical engineer. $\mathrm{He}$ is currently working in Universiti Tunku Abdul Rahman (UTAR) as a lecturer and pursuing his $\mathrm{PhD}$ degree in Engineering. His research interest includes optoelectronics and antenna design. 